\title{
An Application of Variant Fountain Theorems to a Class of Impulsive Differential Equations with Dirichlet Boundary Value Condition
}

\author{
Liu Yang \\ Department of Mathematics, Hengyang Normal University, Hengyang, Hunan 421008, China \\ Correspondence should be addressed to Liu Yang; yangliu19731974@yahoo.com.cn
}

Received 12 February 2014; Accepted 7 April 2014; Published 29 April 2014

Academic Editor: Donal O’Regan

Copyright (C) 2014 Liu Yang. This is an open access article distributed under the Creative Commons Attribution License, which permits unrestricted use, distribution, and reproduction in any medium, provided the original work is properly cited.

We consider the existence of infinitely many classical solutions to a class of impulsive differential equations with Dirichlet boundary value condition. Our main tools are based on variant fountain theorems and variational method. We study the case in which the nonlinearity is sublinear. Some recent results are extended and improved.

\section{Introduction}

Consider the following Dirichlet boundary value problem of impulsive differential equations:

$$
\begin{gathered}
-u^{\prime \prime}\left(t_{0}\right)+g(t) u(t)=f(t, u(t)), \quad \text { a.e. } t \in[0, T], \\
\Delta\left(u^{\prime}\left(t_{j}\right)\right)=I_{j}\left(u\left(t_{j}\right)\right), \quad j=1,2, \ldots, p, \\
u(0)=u(T)=0,
\end{gathered}
$$

where $0=t_{0}<t_{1}<t_{2}<\cdots<t_{p}<t_{p+1}=T, \Delta\left(u^{\prime}\left(t_{j}\right)\right)=$ $u^{\prime}\left(t_{j}^{+}\right)-u^{\prime}\left(t_{j}^{-}\right)=\lim _{s \rightarrow t_{j}^{+}} u^{\prime}(s)-\lim _{s \rightarrow t_{j}^{-}} u^{\prime}(s), g \in L^{\infty}[0, T]$, $f:[0, T] \times \mathbb{R} \rightarrow \mathbb{R}$ is continuous, and $I_{j}: \mathbb{R} \rightarrow \mathbb{R}, 1 \leq j \leq p$ are continuous.

Since impulsive differential equations can describe many evolution processes in which their states are changed abruptly at certain moments of time, they play an important role in applications, such as in control theory, optimization theory, biology, and some physics or mechanics problem; see [1$5]$. For general theory of impulsive differential equations, we refer the readers to the monographs as $[6,7]$. The existence and multiplicity of solutions to impulsive differential equations with boundary value condition have been obtained by using fixed point theorems and upper and lower solutions method; see [8-12] and references therein. Recently, some authors creatively applied variational method to deal with impulsive problems and obtained some new results; see [1318]. For general theory of variational method, we refer the readers to the monographs as $[19,20]$. More precisely, Nieto and O'Regan [13] studied Dirichlet problem as follows:

$$
\begin{gathered}
-\ddot{u}(t)+\lambda u(t)=f(t, u(t)), \quad t \neq t_{j}, \\
\Delta \dot{u}\left(t_{j}\right)=I_{j}\left(u\left(t_{j}\right)\right), \quad j=1,2, \ldots, p, \\
u(0)=u(T)=0 .
\end{gathered}
$$

For the sublinear case, they obtained the following result.

Lemma 1 (See [13]). Assume that the following conditions are satisfied.

(1) There exist $a, b>0$ and $\gamma \in[0,1)$ such that

$|f(t, u)| \leq a+b|u|^{\gamma} \quad$ for every $(t, u) \in[0, T] \times \mathbb{R}$.

(2) There exist $a_{j}, b_{j}>0$ and $\gamma_{j} \in[0,1)(j=1,2, \ldots, p)$ such that

$$
\left|I_{j}(u)\right| \leq a_{j}+b_{j}^{\gamma_{j}} \quad \text { for every } u \in \mathbb{R} .
$$

Then Problem (2) has at least one solution. 
For superlinear case, Zhang and Yuan [15] obtained the existence of one and infinitely many solutions for Problem (2) under the well-known Ambrosetti-Rabinowitz condition; that is, there exists $\theta>2$ such that

$$
0<\theta F(t, u) \leq f(t, u) u, \quad \forall u \in \mathbb{R} \backslash\{0\}, t \in[0, T],
$$

where $F$ is a primitive function of $f$. Soon after, Zhou and $\mathrm{Li}$ [16] obtained the existence of infinitely many solutions for Problem (1) under the weaker condition; there exist $\theta>2$ and $R>0$ such that

$$
0<\theta F(t, u) \leq f(t, u) u, \quad|u| \geq R, \quad \forall t \in[0, T] .
$$

Recently, Sun and Chen studied the existence of infinitely many solutions for Problem (1) with superlinear nonlinearity $f$ which is not satisfied (5) or (6). In addition, they also studied the case where the nonlinearity is asymptotically linear.

Motivated by the above facts, in this paper, our aim is to study the existence of infinitely many solutions for Problem (1) with nonlinearity $f$ which is sublinear. To the best of our knowledge, there are few papers concerned with this. For sublinear case, Nieto and O'Regan [13] only obtain the existence of at least one solution.

We make the following assumptions:

$\left(H_{1}\right) I_{j}(1 \leq j \leq p)$ are odd and satisfy

$$
\frac{1}{2} I_{j}(u) u-\int_{0}^{u} I_{j}(s) d s \geq 0, \quad \int_{0}^{u} I_{j}(s) d s \geq 0
$$

for all $u \in \mathbb{R}$.

$\left(H_{2}\right)$ For any $j \in\{1,2, \ldots, p\}$, there exist constants $b_{j}>0$ and $\gamma_{j} \geq 1$ such that

$$
\left|I_{j}(u)\right| \leq b_{j}|u|^{\gamma_{j}}
$$

for all $u \in \mathbb{R}$.

$\left(H_{3}\right)$ There exist constants $R_{0}>0, d>0$ and $\gamma \geq 1$ such that

$f(t, u) u \geq 0, \quad F(t, u)-\frac{1}{2} f(t, u) u \geq d|u|^{\gamma}$,

for every $t \in[0, T]$ and $u \in \mathbb{R}$ with $|u| \geq R_{0}$, where $F(t, u)=\int_{0}^{u} f(t, s) d s$. Moreover, $F(t, u) \geq 0$ for all $t \in[0, T]$ and $\in \mathbb{R}$.

$\left(H_{4}\right)$ There exist constants $\mu \in(1,2)$ and $C_{1}>0$ such that $|f(t, u)| \leq C_{1}\left(1+|u|^{\mu-1}\right)$. In what follows, $C_{i}$, $i=1,2, \ldots$ denote positive constants.

$\left(H_{5}\right)$ there exist constants $R_{1}>0, C_{2}>0, \delta \in[1,2)$ such that

$$
F(t, u) \geq C_{2}|u|^{\delta}
$$

for every $t \in[0, T]$ and $|u| \leq R_{1}$.

$\left(H_{6}\right) F(t, u)$ is even in $u$, that is, $F(t,-u)=F(t, u)$.
Theorem 2. Assume that $\left(H_{1}\right)-\left(H_{6}\right)$ are satisfied. Then Problem (1) has infinitely many classical solutions.

Remark 3. For the definition of classical solution, we refer readers to paper $[17,18]$. By $\left(H_{1}\right)$ and $\left(H_{2}\right), I_{j}(j=1,2, \ldots, p)$ are not sublinear as those in [13-18]. We note that there are functions $I_{j}(j=1,2, \ldots, p)$ and $f$ which satisfy the conditions of Theorem 2 but do not satisfy the conditions in references we mentioned above. For example, let

$$
\begin{gathered}
I_{j}(s)=s^{3}, \quad j=1,2, \ldots, p, \\
F(t, u)= \begin{cases}\frac{|u|^{3 / 2},}{(3|u|-1)} & |u|<1,\end{cases} \\
f(t, u)= \begin{cases}\frac{3}{2}, & u \geq 1, \\
\frac{3}{2}|u|^{1 / 2}, & |u|<1, \\
-\frac{3}{2}, & u \leq-1 .\end{cases}
\end{gathered}
$$

If we choose $\gamma_{j}=3, R_{0}=1, d=1 / 4, \delta=3 / 2, \gamma=1$, and $\mu=$ $3 / 2$, then it is easy to check that the conditions in Theorem 2 are satisfied.

The organization of this paper is as follows. In Section 2, we shall give some lemmas and some preliminary results. In Section 3, the proofs of the main results are given.

\section{Preliminaries}

In order to prove our main results, we recall the variant fountain theorem. Let $E$ be a Banach space with the norm $\|\cdot\|$ and $E=\overline{\bigoplus_{j=1}^{k} X_{j}}$ with $\operatorname{dim} X_{j}<\infty$ for any $j \in \mathbb{N}$. Set $Y_{k}=\bigoplus_{j=1}^{k} X_{j}, Z_{k}=\bar{\bigoplus}_{j=k}^{\infty} X_{j}$. Consider the following $C^{1}$ functional $I_{\lambda}: E \rightarrow \mathbb{R}$ defined by

$$
I_{\lambda}(u)=A(u)-\lambda B(u), \quad \lambda \in[1,2] .
$$

Lemma 4 (see [21]). Suppose that the functional $I_{\lambda}(u)$ defined above satisfies the following.

(C1) $I_{\lambda}$ maps bounded sets to bounded sets uniformly for $\lambda \in[1,2]$. Furthermore, $I_{\lambda}(-u)=I_{\lambda}(u)$ for all $(\lambda, u) \in$ $[1,2] \times E$.

$(C 2) B(u) \geq 0 ; B(u) \rightarrow \infty$ as $\|u\| \rightarrow \infty$ on any finite dimensional subspace of $E$.

(C3) There exist $\rho_{k}>r_{k}>0$ such that

$$
a_{k}(\lambda):=\inf _{u \in Z_{k},\|u\|=\rho_{k}} I_{\lambda}(u) \geq 0>b_{k}(\lambda):=\max _{u \in Y_{k},\|u\|=r_{k}} I_{\lambda}(u)
$$

for all $\lambda \in[1,2]$ and $d_{k}(\lambda):=\inf _{u \in Z_{k}\|u\| \leq \rho_{k}} I_{\lambda}(u) \rightarrow 0$ as $k \rightarrow \infty$ uniformly for $\lambda \in[1,2]$. Then there exist $\lambda_{n} \rightarrow$ 
$1, u_{\lambda_{n}} \in Y_{n}$ such that $I_{\lambda_{n}}^{\prime} \mid Y_{n}\left(u\left(\lambda_{n}\right)\right)=0, I_{\lambda_{n}}\left(u\left(\lambda_{n}\right)\right) \rightarrow$ $c_{k} \in\left[d_{k}(2), b_{k}(1)\right]$ as $n \rightarrow \infty$. In particular, if $\left\{u\left(\lambda_{n}\right)\right\}$ has a convergent subsequence for every $k$, then $I_{1}$ has infinitely many nontrivial critical points $\left\{u_{n}\right\} \subset E \backslash\{0\}$ satisfying $I_{1}\left(u_{k}\right) \rightarrow 0^{-}$ as $k \rightarrow \infty$.

In the Sobolev space $E:=H_{0}^{1}(0, T)$, consider the inner product

$$
(u, v)_{0}=\int_{0}^{T} u^{\prime}(t) v^{\prime}(t) d t
$$

inducing the norm

$$
\|u\|_{0}=\left(\int_{0}^{T}\left|u^{\prime}(t)\right|^{2} d t\right)^{1 / 2}
$$

for any $u, v \in E$. Since $E$ is compactly embedded in $L^{s}([0, T])$ with norm $|u|_{s}=\left(\int_{0}^{T}|u(t)|^{s} d t\right)^{1 / 2}$ for $s \in[2,+\infty]$, as in $[18,22]$, we know that the eigenvalues of operator $S=-\left(d^{2} / d t^{2}\right)+g$ with the Dirichlet boundary conditions are numbered by $\lambda_{1} \leq \lambda_{2} \leq \cdots \leq \lambda_{n} \leq \cdots \rightarrow$ $\infty$ (counted in their multiplicities) and a corresponding system of eigenfunctions $\left\{e_{j}\right\}$, which forms the completely orthogonal basis in $L^{2}([0, T])$. Assume $\lambda_{1}, \ldots, \lambda_{n^{-}}<0$, $\lambda_{n^{-}+1}=\cdots=\lambda_{n^{0}}=0$ and let $E^{-}=\operatorname{span}\left\{e_{1}, e_{2}, \ldots, e_{n^{-}}\right\}, E^{0}=$ $\operatorname{span}\left\{e_{n^{-}+1}, \ldots, e_{n^{0}}\right\}$, and $E^{+}=\operatorname{span}\left\{e_{n^{0}+1}, \ldots,\right\}$. Then $E=$ $E^{-} \oplus E^{0} \oplus E^{+}$. We introduce on $E$ the following product $(u, v)=$ $\left(\left|S^{1 / 2}\right| u,\left|S^{1 / 2}\right| v\right)_{L^{2}}+\left(u^{0}, v^{0}\right)_{L^{2}}$ and norm $\|u\|=(u, u)^{1 / 2}$, where $u=u^{-}+u^{0}+u^{+}, v=v^{-}+v^{0}+v^{+} \in E^{-} \oplus E^{0} \oplus E^{+}$. Then $\|\cdot\|$ and $\|\cdot\|_{0}$ are equivalent. By the Soblev imbedding theorem, $E$ is compactly embedded in $C[0, T]$, and there exists $C_{3}>0$ such that

$$
\|u\|_{\infty} \leq C_{3}\|u\|
$$

where

$$
\|u\|_{\infty}=\max _{t \in[0, T]}|u(t)|
$$

Define a functional $\varphi$ on $E$ by

$$
\begin{aligned}
\varphi(u)= & \frac{1}{2} \int_{0}^{T}\left(\left|u^{\prime}\right|^{2}+g(t) u^{2}\right) d t+\sum_{j=1}^{p} \int_{0}^{u\left(t_{j}\right)} I_{j}(s) d s \\
& -\int_{0}^{T} F(t, u) d t \\
= & \frac{1}{2}\left(\left\|u^{+}\right\|^{2}-\left\|u^{-}\right\|^{2}\right)+\sum_{j=1}^{p} \int_{0}^{u\left(t_{j}\right)} I_{j}(s) d s \\
& -\int_{0}^{T} F(t, u) d t
\end{aligned}
$$

where $u=u^{-}+u^{0}+u^{+} \in E$ with $u^{-} \in E^{-}, u^{0} \in E^{0}, u^{+} \in E^{+}$. By the conditions of Theorem 2 , we know that $\varphi$ is continuously differentiable and

$$
\begin{aligned}
& \left\langle\varphi^{\prime}(u), v\right\rangle \\
& =\int_{0}^{T}\left(u^{\prime} v^{\prime}+g(t) u v\right) d t+\sum_{j=1}^{p} I_{j}\left(u\left(t_{j}\right)\right) v\left(t_{j}\right) \\
& \quad-\int_{0}^{T} f(t, u) v d t \\
& =\left(u^{+}-u^{-}, v\right)+\sum_{j=1}^{p} I_{j}\left(u\left(t_{j}\right)\right) v\left(t_{j}\right)-\int_{0}^{T} f(t, u) v d t,
\end{aligned}
$$

for any $u, v \in E$.

Like for Lemma 2.4 in [17], one can prove that the critical points of the functional $\varphi$ are the classical solutions for Problem (1).

\section{Proofs of the Main Results}

Now we define a class of functionals on $E$ by

$$
\begin{aligned}
\varphi_{\lambda}(u):= & \frac{1}{2}\left\|u^{+}\right\|^{2}+\sum_{j=1}^{p} \int_{0}^{u\left(t_{j}\right)} I_{j}(s) d s \\
& -\lambda\left(\frac{1}{2}\left\|u^{-}\right\|^{2}+\int_{0}^{T} F(t, u) d t\right) \\
:= & A(u)-\lambda B(u), \quad \lambda \in[1,2],
\end{aligned}
$$

where $u^{-} \in E^{-}, u^{+} \in E^{+}$. Denote by $X_{j}:=\operatorname{span}\left\{e_{j}\right\}, j \in \mathbb{N}$. Clearly, we see that $\varphi_{\lambda} \in C^{1}(E, \mathbb{R})$ for all $\lambda \in[1,2]$ and the critical points of $\varphi_{1}$ correspond to the solutions to Problem (1).

Lemma 5. Let $\left(\mathrm{H}_{3}\right)$ be satisfied. Then $B(u) \geq 0$. Furthermore, $B(u) \rightarrow \infty$ as $\|u\| \rightarrow \infty$ on any finite dimensional subspace of $E$.

Proof. Evidently, $B(u) \geq 0$ follows by the definition of the functional $B$ and $\left(H_{3}\right)$. Now we claim that for any finite dimensional subspace of $F \subset E$, there exists $\varepsilon>0$ such that

$$
\operatorname{meas}(\{t \in[0, T]:|u(t)| \geq \varepsilon\|u\|\}) \geq \varepsilon, \forall u \in F \backslash\{0\} \text {, }
$$

where meas denotes the Lebesgue measure in $\mathbb{R}$. that

Otherwise, for any $n \in \mathbb{N}$, there exists $u_{n} \in F \backslash\{0\}$ such

$$
\text { meas }\left(\left\{t \in[0, T]:\left|u_{n}(t)\right| \geq \frac{1}{n}\|u\|\right\}\right)<\frac{1}{n} \text {. }
$$

Let $v_{n}=u_{n} /\left\|u_{n}\right\| \in F \backslash\{0\}$ for all $n \in \mathbb{N}$. Then $\left\|v_{n}\right\|=1$ and

$$
\operatorname{meas}\left(\left\{t \in[0, T]:\left|u_{n}(t)\right| \geq \frac{1}{n}\right\}\right)<\frac{1}{n}, \quad \forall n \in \mathbb{N} \text {. }
$$


Since $\operatorname{dim} F<\infty$, it follows from the compactness of the unit sphere of $F$ that there exists a subsequence, say $\left\{v_{n}\right\}$, such that $v_{n} \rightarrow v_{0}$ in $F$. Hence, we have $\left\|v_{0}\right\|=1$. By the equivalence of the norms on the finite dimensional space $F$, we have $v_{n} \rightarrow$ $v_{0}$ in $L^{2}[0, T]$, that is,

$$
\int_{0}^{T}\left|v_{n}-v_{0}\right|^{2} d t \longrightarrow 0 \quad \text { as } n \longrightarrow \infty
$$

Thus there exist $\xi_{1}, \xi_{2}>0$ such that

$$
\text { meas }\left\{t \in[0, T]:\left|v_{0}(t)\right| \geq \xi_{1}\right\} \geq \xi_{2} \text {. }
$$

In fact, if not, we have

$$
\text { meas }\left\{t \in[0, T]:\left|v_{0}(t)\right| \geq \frac{1}{n}\right\}=0, \quad \forall n \in \mathbb{N} \text {. }
$$

This implies that

$$
0<\int_{0}^{T}\left|v_{0}\right|^{2} d t<\frac{1}{n^{2}} T \longrightarrow 0, \quad \text { as } n \longrightarrow \infty,
$$

which gives a contradiction.

Now let

$$
\begin{aligned}
& \Omega_{0}=\left\{t \in[0, T]:\left|v_{0}(t)\right| \geq \xi_{1}\right\}, \\
& \Omega_{n}=\left\{t \in[0, T]:\left|v_{n}(t)\right|<\frac{1}{n}\right\},
\end{aligned}
$$

and $\Omega_{n}^{\perp}=[0, T] \backslash \Omega_{n}$. We have

$$
\text { meas }\left(\Omega_{n} \cap \Omega_{0}\right) \geq \operatorname{meas}\left(\Omega_{0}\right)-\operatorname{meas}\left(\Omega_{n}^{\perp} \cap \Omega_{0}\right) \geq \xi_{2}-\frac{1}{n}
$$

for all positive integer $n$. Let $n$ be large enough such that $\xi_{2}-$ $(1 / n) \geq(1 / 2) \xi_{2}$ and $\xi_{1}-(1 / n) \geq(1 / 2) \xi_{1}$. Then we have

$$
\left|v_{n}(t)-v_{0}(t)\right|^{2} \geq\left(\xi_{1}-\frac{1}{n}\right)^{2} \geq \frac{1}{4} \xi_{1}^{2}, \quad \forall t \in \Omega_{n} \cap \Omega_{0} .
$$

This implies that

$$
\begin{aligned}
\int_{0}^{T}\left|v_{n}(t)-v_{0}(t)\right|^{2} d t & \\
\quad & \geq \int_{\Omega_{n} \cap \Omega_{0}}\left|v_{n}(t)-v_{0}(t)\right|^{2} d t \\
& \geq \frac{1}{4} \xi_{1}^{2} \operatorname{meas}\left(\Omega_{n} \cap \Omega_{0}\right) \\
& \geq \frac{1}{4} \xi_{1}^{2}\left(\xi_{2}-\frac{1}{n}\right) \geq \frac{1}{8} \xi_{1}^{2} \xi_{2}>0
\end{aligned}
$$

for all large $n$, which is a contradiction with (24). For the $\varepsilon$ given in (21), let

$$
\Lambda_{u}=\{t \in[0, T]:|u(t)| \geq \varepsilon\|u\|\}, \quad \forall u \in F \backslash\{0\} .
$$

Then

$$
\operatorname{meas}\left(\Lambda_{u}\right) \geq \varepsilon, \quad \forall u \in F \backslash\{0\}
$$

Observing that for any $u \in F$ with $\|u\| \geq R_{0} / \varepsilon$, the following inequality holds

$$
|u(t)| \geq R_{0}, \quad \forall t \in \Lambda_{u} .
$$

Combining (34) and $\left(H_{3}\right)$, for any $u \in F$ with $\|u\| \geq R_{0} / \varepsilon$, we have

$$
\begin{aligned}
B(u) & =\frac{1}{2}\left\|u^{-}\right\|^{2}+\int_{0}^{T} F(t, u) d t \\
& \geq \int_{\Lambda_{u}} F(t, u) d t \\
& \geq \int_{\Lambda_{u}} d|u|^{\gamma} d t \\
& \geq d(\varepsilon\|u\|)^{\gamma} \operatorname{meas}\left(\Lambda_{u}\right) \geq d \varepsilon^{\gamma+1}\|u\|^{\gamma} .
\end{aligned}
$$

This implies $B(u) \rightarrow \infty$ as $\|u\| \rightarrow \infty$ on any finite dimensional subspace of $F \subset E$.

Lemma 6. Assume that $\left(H_{1}\right),\left(H_{4}\right)$ are satisfied. Then there exist a positive integer $k_{0}$ and a sequence $\rho_{k} \rightarrow 0^{+}$as $k \rightarrow \infty$ such that

$$
a_{k}(\lambda):=\inf _{u \in Z_{k},\|u\|=\rho_{k}} I_{\lambda}(u) \geq 0, \quad \forall k \geq k_{0}
$$

and $d_{k}(\lambda):=\inf _{u \in Z_{k}\|u\| \leq \rho_{k}} I_{\lambda}(u) \rightarrow 0$ as $k \rightarrow \infty$ uniformly for $\lambda \in[1,2]$, where $Z_{k}=\overline{\bigoplus_{j=k}^{\infty} X_{j}}=\overline{\operatorname{span}\left\{e_{k, \cdots}\right\}}$ for all $k \in \mathbb{N}$.

Proof. Note first that $Z_{k} \subset E^{+}$for all $k \geq n^{0}+1$ by definition of $E^{+}$in Section 2 . Thus for any $k \geq n^{0}+1$, by $\left(H_{1}\right),\left(H_{4}\right)$, we have

$$
\begin{aligned}
\varphi_{\lambda}(u) & =\frac{1}{2}\|u\|^{2}+\sum_{j=1}^{p} \int_{0}^{u\left(t_{j}\right)} I_{j}(s) d s-\lambda \int_{0}^{T} F(t, u) d t \\
& \geq \frac{1}{2}\|u\|^{2}-2 C_{1} \int_{0}^{T}\left(|u|+|u|^{\mu}\right) d t \\
& \geq \frac{1}{2}\|u\|^{2}-2 T C_{1}\left(\|u\|_{\infty}+\|u\|_{\infty}^{\mu}\right)
\end{aligned}
$$

for all $(\lambda, u) \in[1,2] \times Z_{k}$. Set $\beta_{k}:=\sup _{u \in Z_{k},\|u\|=1}\|u\|_{\infty}$. Then

$$
\beta_{k} \longrightarrow 0 \text { as } k \longrightarrow \infty \text {. }
$$

Indeed, it is clear that $0<\beta_{k+1} \leq \beta_{k}$, so $\beta_{k} \rightarrow \bar{\beta} \geq 0$ as $k \rightarrow \infty$. For every $k \geq 0$, there exists $u_{k} \in Z_{k}$ such that $\left\|u_{k}\right\|=1$ and $\left\|u_{k}\right\|_{\infty}>\beta_{k} / 2$. By the definition of $Z_{k}, u_{k} \rightarrow 0$ in $E$. Then this implies that $u_{k} \rightarrow 0$ in $C[0, T]$. Thus we have proved that $\bar{\beta}=0$. Therefore, for any $k \geq n^{0}+1$, the following inequality holds:

$$
\varphi_{\lambda}(u) \geq \frac{1}{2}\|u\|^{2}-2 T C_{1}\left(\beta_{k}\|u\|+\beta_{k}^{\mu}\|u\|^{\mu}\right),
$$

for all $(\lambda, u) \in[1,2] \times Z_{k}$. Let $\rho_{k}=16 T C_{1} \beta_{k}+$ $\left[16 T C_{1} \beta_{k}^{\mu}\right]^{1 /(2-\mu)}$. Then by (38), we have $\rho_{k} \rightarrow 0^{+}$as $k \rightarrow$ $\infty$. Hence, by (39), straightforward computation shows that 
$a_{k}(\lambda) \geq \rho_{k}^{2} / 4>0$. Furthermore, for any $k \geq k_{0}$ and $u \in Z_{k}$ with $\|u\| \leq \rho_{k}$, we have

$$
\varphi_{\lambda}(u) \geq-2 T C_{1}\left(\beta_{k}\|u\|+\beta_{k}^{\mu}\|u\|^{\mu}\right) .
$$

Clearly, we see that $F(t, 0)=0$ by the definition of $F$ and $\varphi_{\lambda}(0)=0$. Therefore,

$$
0 \geq \inf _{u \in Z_{k},\|u\| \leq \rho_{k}} \varphi_{\lambda}(u) \geq-2 T C_{1}\left(\beta_{k}\|u\|+\beta_{k}^{\mu}\|u\|^{\mu}\right)
$$

Combining (38) and (41), we have $d_{k}(\lambda)$ := $\inf _{u \in Z_{k}\|u\| \leq \rho_{k}} I_{\lambda}(u) \rightarrow 0$ as $k \rightarrow \infty$ uniformly for $\lambda \in[1,2]$.

Lemma 7. Let $\left(\mathrm{H}_{2}\right),\left(\mathrm{H}_{5}\right)$ be satisfied. Then for the sequence $\left\{\rho_{k}\right\}_{k \in \mathbb{N}}$ obtained in Lemma 6, there exist $0<r_{k}<\rho_{k}$ for all $k \in \mathbb{N}$ such that

$$
b_{k}(\lambda):=\max _{u \in Y_{k},\|u\|=r_{k}} I_{\lambda}(u)<0, \quad \forall k \in \mathbb{N}
$$

where $Y_{k}=\bigoplus_{j=1}^{k} X_{j}=\operatorname{span}\left\{e_{1}, e_{2}, \ldots, e_{k}\right\}$ for all $k \in \mathbb{N}$.

Proof. Let $u \in Y_{k}$ with $u=u^{-}+u^{0}+u^{+} \in E=E^{-} \oplus E^{0} \oplus E^{+}$. By (16), for any $u \in Y_{k}$ with $\|u\| \leq R_{1} / C_{3}$, one has $\|u\|_{\infty} \leq R_{1}$. By $\left(H_{5}\right)$, we obtain

$$
\begin{aligned}
\varphi_{\lambda}(u)= & \frac{1}{2}\left\|u^{+}\right\|^{2}+\sum_{j=1}^{p} \int_{0}^{u\left(t_{j}\right)} I_{j}(s) d s \\
& -\lambda\left(\frac{1}{2}\left\|u^{-}\right\|^{2}+\int_{0}^{T} F(t, u) d t\right) \\
\leq & \frac{1}{2}\left\|u^{+}\right\|^{2}+\sum_{j=1}^{p} b_{j}\left|u\left(t_{j}\right)\right|^{\gamma_{j}+1}-C_{2} \int_{0}^{T}|u|^{\delta} d t \\
\leq & \frac{1}{2}\left\|u^{+}\right\|^{2}+\sum_{j=1}^{p} b_{j} C_{3}^{\gamma_{j}+1}\|u\|^{\gamma_{j}+1}-C_{2} C_{4}\|u\|^{\delta}
\end{aligned}
$$

for any $u \in Y_{k}$ with $\|u\| \leq R_{1} / C_{3}$, where the last inequality follows by the equivalence of the norms on the finite dimensional space $Y_{k}$. Since $\delta<2, \gamma_{j} \geq 1(j \in$ $\{1,2, \ldots, p\})$, for $\|u\|=r_{k}$, are small enough, we can get $b_{k}(\lambda):=\max _{u \in Y_{k},\|u\|=r_{k}} I_{\lambda}(u)<0, \forall k \in \mathbb{N}$.

Proof of Theorem 2. By $\left(H_{2}\right),\left(H_{4}\right), \varphi_{\lambda}$ maps bounded sets to bounded sets uniformly for $\lambda \in[1,2]$. Evidently, $\left(H_{1}\right),\left(H_{6}\right)$ imply that $\varphi_{\lambda}(-u)=\varphi_{\lambda}(u)$ for all $(\lambda, u) \in[1,2] \times E$. Thus by Lemma 4 , there exist $\lambda_{n} \rightarrow 1, u_{\lambda_{n}} \in Y_{n}$ such that $\left.\varphi_{\lambda_{n}}^{\prime}\right|_{Y_{n}}\left(u\left(\lambda_{n}\right)\right)=0, \varphi_{\lambda_{n}}\left(u\left(\lambda_{n}\right)\right) \rightarrow c_{k} \in\left[d_{k}(2), b_{k}(1)\right]$ as $n \rightarrow \infty$. For the sake of notational simplicity, in what follows we always set $u_{n}=u_{\lambda_{n}}$ for all $n \in \mathbb{N}$. By $\left(H_{1}\right),\left(H_{3}\right)$, one has

$$
\begin{aligned}
-\varphi_{\lambda_{n}}\left(u_{n}\right)= & \left.\frac{1}{2} \varphi_{\lambda_{n}}^{\prime}\right|_{Y_{n}}\left(u_{n}\right) u_{n}-\varphi_{\lambda_{n}}\left(u_{n}\right) \\
= & \sum_{j=1}^{p}\left[\frac{1}{2} I_{j}\left(u_{n}\left(t_{j}\right)\right) u_{n}\left(t_{j}\right)-\int_{0}^{u_{n}\left(t_{j}\right)} I_{j}(s) d s\right] \\
& +\lambda_{n} \int_{0}^{T}\left[F\left(t, u_{n}\right)-\frac{1}{2} f\left(t, u_{n}\right) u_{n}\right] d t \\
\geq & \lambda_{n} \int_{\Gamma_{n}}\left[F\left(t, u_{n}\right)-\frac{1}{2} f\left(t, u_{n}\right) u_{n}\right] d t-C_{5} \\
\geq & d \lambda_{n} \int_{\Gamma_{n}}\left|u_{n}\right|^{\gamma} d t-C_{5}, \quad n \in \mathbb{N},
\end{aligned}
$$

where $\Gamma_{n}=\left\{t \in[0, T]:\left|u_{n}(t)\right| \geq R_{0}\right\}$ and $C_{5}>0$ is a constant. Hence, there exists a constant $C_{6}>0$ such that $\int_{\Gamma_{n}}\left|u_{n}\right|^{\gamma} d t \leq C_{6}, \forall n \in \mathbb{N}$. On the other hand, we can easily obtain that $\int_{[0, T] \backslash \Gamma_{n}}\left|u_{n}\right|^{\gamma} d t \leq T R_{0}^{\gamma}, \forall n \in \mathbb{N}$. Thus, we have $\int_{0}^{T}\left|u_{n}\right|^{\gamma} d t \leq C_{7}$. In view of the equivalence of any two norms on finite dimensional space $E^{-} \oplus E^{0}$ and (16), we obtain

$$
\begin{aligned}
\left|u_{n}^{-}+u_{n}^{0}\right|_{2}^{2} & =\left(u_{n}^{-}+u_{n}^{0}, u_{n}\right)_{2} \\
& =\left|u_{n}\right|_{\gamma}\left|u_{n}^{-}+u_{n}^{0}\right|_{\gamma^{\prime}} \\
& \leq C_{8}\left|u_{n}^{-}+u_{n}^{0}\right|_{2},
\end{aligned}
$$

where $\gamma^{\prime}=(\gamma /(\gamma-1))\left(\gamma^{\prime}=\infty\right.$ when $\left.\gamma=1\right)$. Therefore, we have

$$
\left|u_{n}^{-}+u_{n}^{0}\right|_{2} \leq C_{8} .
$$

In view of the equivalence of norms on $E^{-} \oplus E^{0}$, we obtain $\left\|u_{n}^{-}+u_{n}^{0}\right\| \leq C_{9}, \forall n \in \mathbb{N}$. Note that

$$
\begin{aligned}
\left\|u_{n}^{+}\right\|^{2}= & 2 \varphi_{\lambda_{n}}\left(u_{n}\right)+\lambda_{n}\left\|u_{n}^{-}\right\|^{2}+2 \lambda_{n} \int_{0}^{T} F\left(t, u_{n}\right) d t \\
& -\sum_{j=1}^{p} \int_{0}^{u_{n}\left(t_{j}\right)} I_{j}(s) d s .
\end{aligned}
$$

Thus

$$
\begin{aligned}
\left\|u_{n}\right\|^{2}= & \left\|u_{n}^{+}\right\|^{2}+\left\|u_{n}^{-}+u_{n}^{0}\right\|^{2} \\
\leq & 2 \varphi_{\lambda_{n}}\left(u_{n}\right)+\lambda_{n}\left\|u_{n}^{-}\right\|^{2}+\left\|u_{n}^{-}+u_{n}^{0}\right\|^{2} \\
& +2 \lambda_{n} \int_{0}^{T} F\left(t, u_{n}\right) d t-\sum_{j=1}^{p} \int_{0}^{u_{n}\left(t_{j}\right)} I_{j}(s) d s \\
\leq & C_{10}+C_{11} \int_{0}^{T}\left[\left|u_{n}\right|+\left|u_{n}\right|^{\mu}\right] \\
\leq & C_{12}+C_{13} T\left[C_{3}\left\|u_{n}\right\|+C_{3}^{\mu}\left\|u_{n}\right\|^{\mu}\right] .
\end{aligned}
$$


Since $\mu<2$, we have $\left\|u_{n}\right\| \leq C_{14}$; that is, $\left\{u_{n}\right\}$ is bounded in $E$. By a standard argument, this yields a critical point $u^{k}$ of $\varphi$ such that $\varphi\left(u^{k}\right) \in\left[d_{k}(2), c_{k}(1)\right]$. Since $d_{k}(2) \rightarrow 0^{-}$as $k \rightarrow \infty$, we can obtain infinitely many critical points.

\section{Conflict of Interests}

The author declares that there is no conflict of interests regarding the publication of this paper.

\section{Acknowledgment}

This work was supported by the National Natural Science Foundation of China (no. 10871206).

\section{References}

[1] R. K. George, A. K. Nandakumaran, and A. Arapostathis, "A note on controllability of impulsive systems," Journal of Mathematical Analysis and Applications, vol. 241, no. 2, pp. 276283, 2000.

[2] T. E. Carter, "Necessary and sufficient conditions for optimal impulsive rendezvous with linear equations of motion," Dynamics and Control, vol. 10, no. 3, pp. 219-227, 2000.

[3] Y. Pei, C. Li, L. Chen, and C. Wang, "Complex dynamics of oneprey multi-predator system with defensive ability of prey and impulsive biological control on predators," Advances in Complex Systems, vol. 8, no. 4, pp. 483-495, 2005.

[4] X. Liu and A. R. Willms, "Impulsive controllability of linear dynamical systems with applications to maneuvers of spacecraft," Mathematical Problems in Engineering, vol. 2, no. 4, pp. 277-299, 1996.

[5] S. Gao, L. Chen, J. J. Nieto, and A. Torres, "Analysis of a delayed epidemic model with pulse vaccination and saturation incidence," Vaccine, vol. 24, no. 35-36, pp. 6037-6045, 2006.

[6] V. Lakshmikantham, D. D. Bainnov, and P. S. Simeonov, Theory of Impulsive Differential Equations, World Scientific, Singapore, 1989.

[7] W. M. Haddad, V. Chellaboina, S. G. Nersesov, and G. Sergey, Impulsive and Hybrid Dynamical Systems: Stability, Dissipativity, and Control, Princeton University Press, Princeton, NJ, USA, 2006.

[8] R. P. Agarwal and D. O’Regan, “A multiplicity result for second order impulsive differential equations via the Leggett Williams fixed point theorem," Applied Mathematics and Computation, vol. 161, no. 2, pp. 433-439, 2005.

[9] H. Wang and H. Chen, "Boundary value problem for secondorder impulsive functional differential equations," Applied Mathematics and Computation, vol. 191, no. 2, pp. 582-591, 2007.

[10] J. Chen, C. C. Tisdell, and R. Yuan, "On the solvability of periodic boundary value problems with impulse," Journal of Mathematical Analysis and Applications, vol. 331, no. 2, pp. 902912, 2007.

[11] J. Li, J. J. Nieto, and J. Shen, "Impulsive periodic boundary value problems of first-order differential equations," Journal of Mathematical Analysis and Applications, vol. 325, no. 1, pp. 226236, 2007.

[12] E. Hernández M, H. R. Henríquez, and M. A. McKibben, "Existence results for abstract impulsive second-order neutral functional differential equations," Nonlinear Analysis: Theory, Methods \& Applications, vol. 70, no. 7, pp. 2736-2751, 2009.

[13] J. J. Nieto and D. O’Regan, "Variational approach to impulsive differential equations," Nonlinear Analysis: Real World Applications, vol. 10, no. 2, pp. 680-690, 2009.

[14] Y. Tian and W. Ge, "Applications of variational methods to boundary-value problem for impulsive differential equations," Proceedings of the Edinburgh Mathematical Society, vol. 51, no. 2, pp. 509-527, 2008.

[15] Z. Zhang and R. Yuan, "An application of variational methods to Dirichlet boundary value problem with impulses," Nonlinear Analysis: Real World Applications, vol. 11, no. 1, pp. 155-162, 2010.

[16] J. Zhou and Y. Li, "Existence and multiplicity of solutions for some Dirichlet problems with impulsive effects," Nonlinear Analysis: Theory, Methods \& Applications, vol. 71, no. 7-8, pp. 2856-2865, 2009.

[17] J. Sun and H. Chen, "Variational method to the impulsive equation with neumann boundary conditions," Boundary Value Problems, vol. 2009, Article ID 316812, 17 pages, 2009.

[18] J. Sun and H. Chen, "Multiplicity of solutions for a class of impulsive differential equations with Dirichlet boundary conditions via variant fountain theorems," Nonlinear Analysis: Real World Applications, vol. 11, no. 5, pp. 4062-4071, 2010.

[19] J. Mawhin and M. Willem, Critical Point Theory and Hamiltonian Systems, Springer, Berlin, Germany, 1989.

[20] P. H. Rabinowitz, Minimax Methods in Critical Point Theory with Applications to Differential Equations, vol. 65 of Conference Board of the Mathematical Sciences Regional Conference Series in Mathematics, American Mathematical Society, Providence, RI, USA, 1986

[21] W. Zou, "Variant fountain theorems and their applications," Manuscripta Mathematica, vol. 104, no. 3, pp. 343-358, 2001.

[22] D. Yanheng, "Existence and multiplicity results for homoclinic solutions to a class of Hamiltonian systems," Nonlinear Analysis: Theory, Methods \& Applications, vol. 25, no. 11, pp. 1095-1113, 1995. 


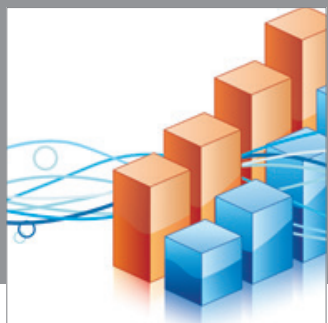

Advances in

Operations Research

mansans

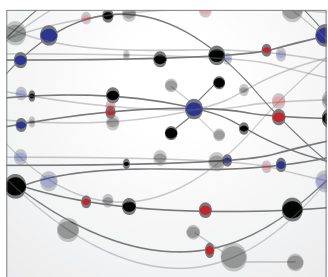

The Scientific World Journal
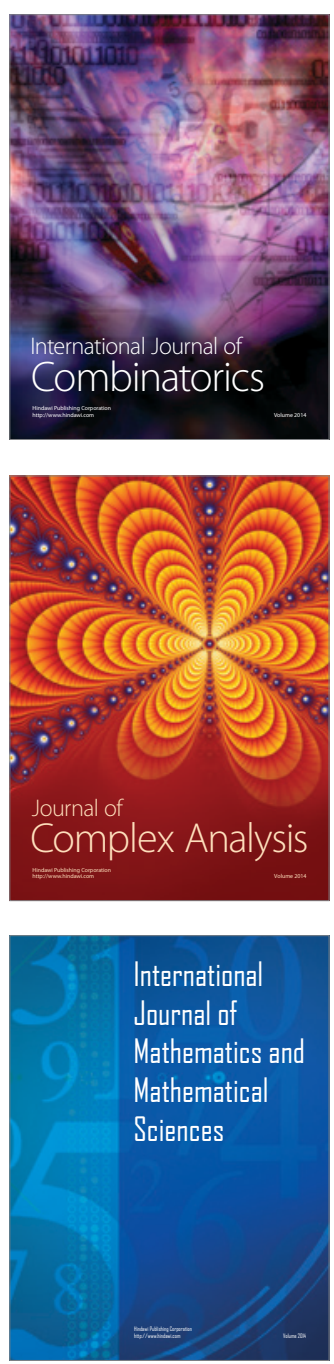
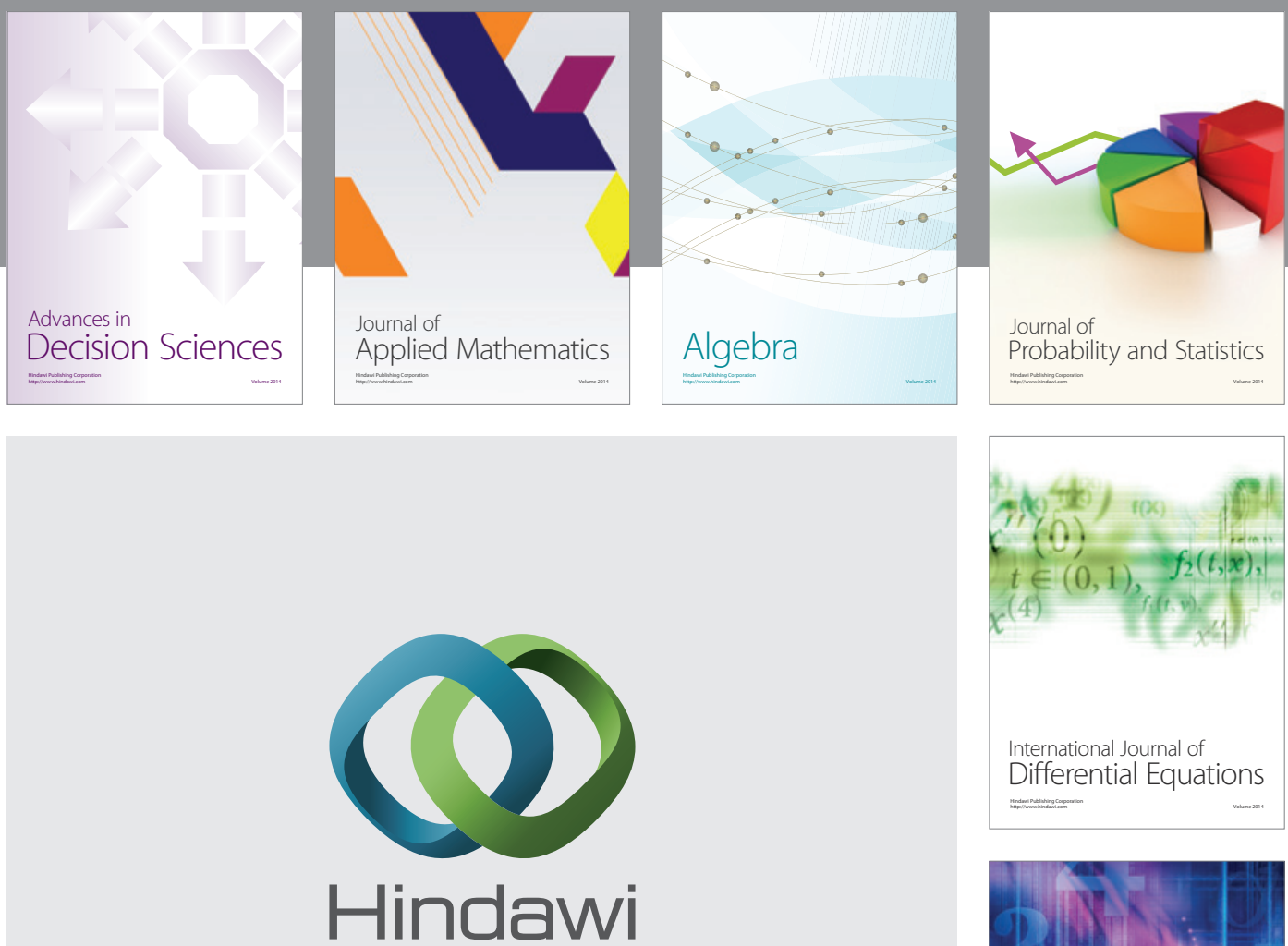

Submit your manuscripts at http://www.hindawi.com
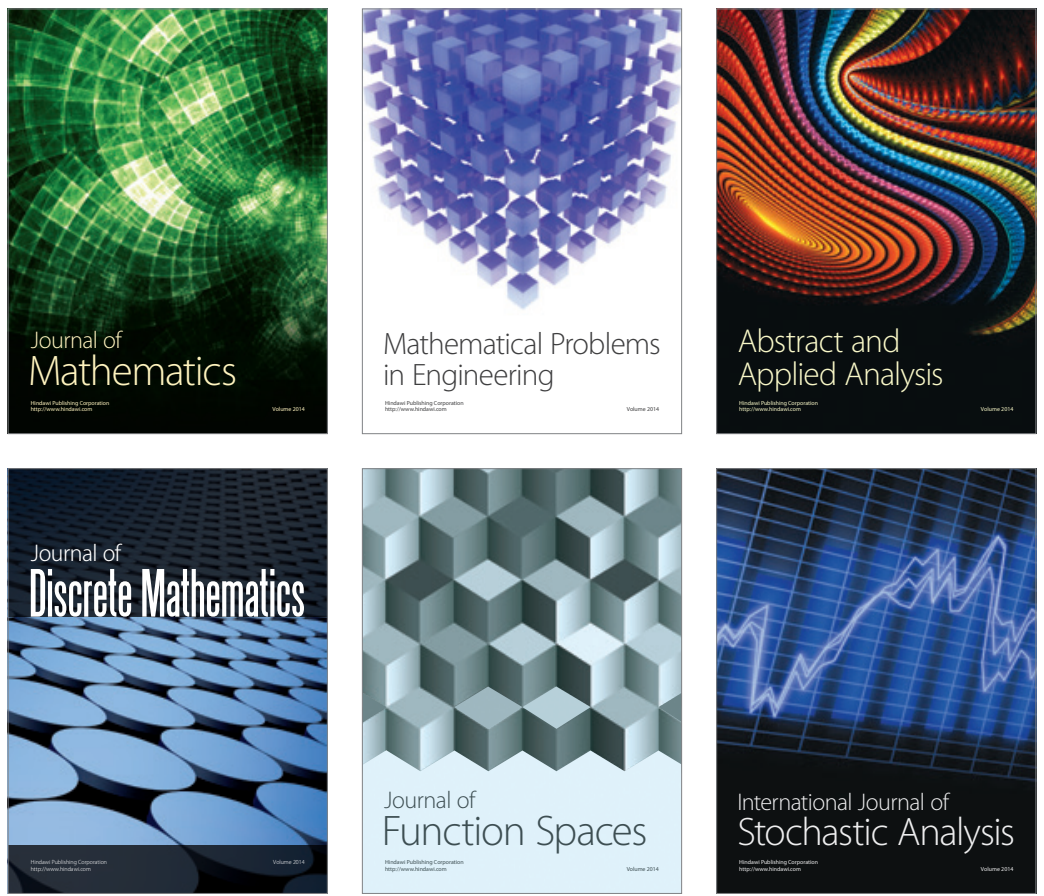

Journal of

Function Spaces

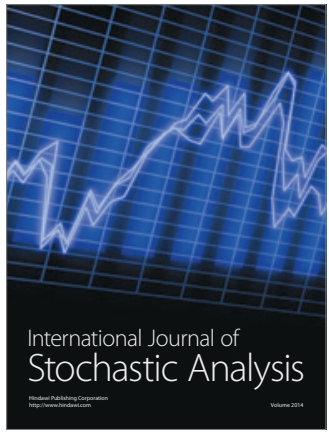

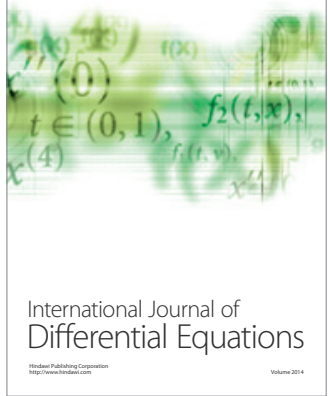
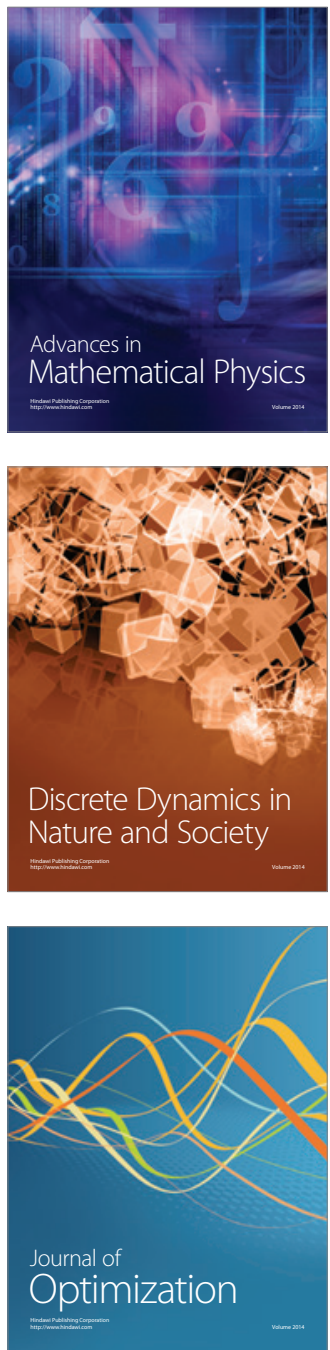